\section{Endoscopic Treatment of Esophageal Carcinosarcoma: Report of a Case}

A 73-year-old man was admitted to our institution because of dysphagia. Upper gastrointestinal endoscopy revealed a bulky intraluminal polypoid lesion in the cervical esophagus (Figure 1). Histological results indicated a sarcoma, and computed tomography (CT) scan revealed a tumor limited to the esophageal wall. The patient could not undergo surgery because of cardiac dysfunction, and we decided to perform an endoscopic mucosal resection (EMR). Using a sclerotherapy needle, we injected serum and adrenaline $(1 / 10)$ into the lower part of the tumor in order to separate the mucosal and submucosal layers from the muscularis tissues. Then we resected the bulky pedunculated tumor $(3 \times 7 \mathrm{~cm}$; Figure 2$)$ using a polypectomy loop. Histological and immunohistochemical studies confirmed that the tumor was a sarcoma (myofibroblastic phenotype). At 1 month later, endoscopy showed a discrete area of thickness $2 \mathrm{~mm}$ that corresponded to the EMR scar. Biopsy results pointed to an undifferentiated squamous cell carcinoma. In fact the tumor was mixed (carcinosarcoma), with both carcinomatous and sarcomatous components being present. A cervical thoracic and abdominal CT scan showed only a small mediastinal lymph node. There were no cervical lymph nodes, neither was there abnormal thickening of the esophageal wall, mediastinal infiltration, or hepatic metastasis. The patient underwent comple-

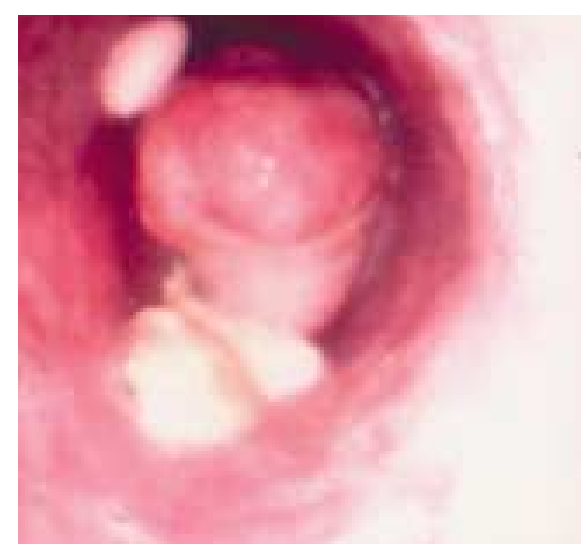

Figure 1 A bulky intraluminal polypoid lesion in the cervical esophagus caused dysphagia. A tablet and remnants of food can be seen above the tumor. mentary chemoradiotherapy. Radiotherapy consisted of a dose of $60 \mathrm{~Gy}$ delivered to the superior third of the esophagus (2 Gy for five sessions per week for 6 weeks), and the chemotherapy was cisplatyl $\left(20 \mathrm{mg} / \mathrm{m}^{2}\right.$ once a week over 6 weeks). Clinical and biological tolerance of this treatment were excellent. At 2 months after the end of this treatment, endoscopy and endoscopic ultrasonography (EUS) showed a normal esophageal wall, and follow-up CT scan confirmed complete resolution. Since then, we have followed up the patient regularly with clinical examination, CT scan, and EUS. Complete remission has always been found, and there is no sign of relapse at 2.5 years after the diagnosis. Carcinosarcoma of the esophagus is a rare malignant neoplasm, predominantly affecting men in their seventh decade of life [1]. The more favorable prognosis associated with carcinosarcoma compared with other esophageal neoplasms has been attributed to the early onset of symptoms which leads to prompt diagnosis, and a lower propensity for tumor invasion [1]. Occurrence of this type of tumor is rather unusual in a patient who has never been previously treated with esophageal radiotherapy or surgery. To our knowledge this is the second case of esophageal carcinosarcoma to be successfully treated endoscopically [2], and the first to be treated by EMR $[3,4]$. This unusual approach was undertaken only be-

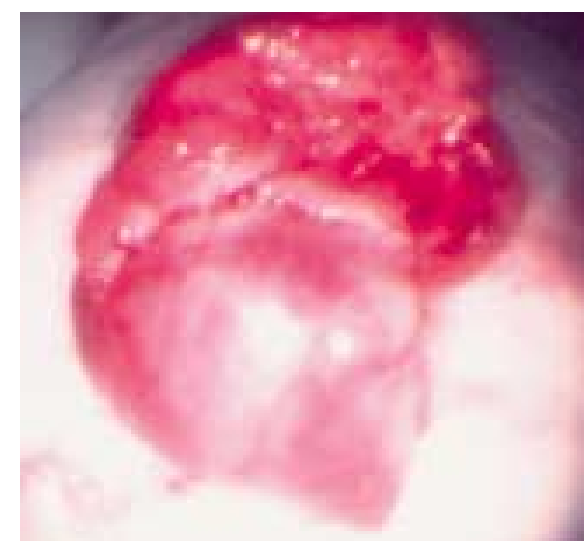

Figure 2 The bulky pedunculated carcinosarcoma $(3 \times 7 \mathrm{~cm})$ after resection using a polypectomy loop. cause the patient's general condition precluded esophagectomy with lymph node dissection which is still the "gold standard" treatment [5,6]. Similarly, chemoradiotherapy alone could not be considered because of the tumoral volume and dysphagia. In conclusion, EMR associated with other treatments is an interesting alternative to surgery for a patient with a large pedunculated esophageal tumor who cannot undergo operation.

\section{Pesenti ${ }^{1}$, E. Bories ${ }^{1}$, C. Danisi ${ }^{1}$, G. Monges ${ }^{2}$, M. Giovannini ${ }^{1}$ \\ ${ }^{1}$ Department of Endoscopy, Paoli \\ Calmettes Institute, Marseille, France \\ 2 Department of Anatomopathology, Paoli \\ Calmettes Institute, Marseille, France.}

References

${ }^{1}$ Ziauddin MF, Rodriguez HE, Quiros ED et al. Carcinosarcoma of the esophagus pattern of recurrence. Dig Surg 2001; 18: 216- 218

2 Takeda T, Konda Y, Miyamoto M et al. A case of esophageal carcinosarcoma treated effectively with endoscopic polypectomy and irradiation. Nippon Shokakibyo Gakkai Zasshi 1987; 84: 286-290

${ }^{3}$ Shim CS. Endoscopic mucosal resection: an overview of the value of different techniques. Endoscopy 2001; 33: 271 - 275

${ }^{4}$ Leiper K, Morris AI. State of the art review. Treatment of oesophagogastric tumours. Endoscopy 2002; 34: 139-145

${ }^{5}$ Iyomasa S, Kato H, Tachimori Y et al. Carcinosarcoma of the esophagus: a 20-case study. Jpn J Clin Oncol 1990; 20: 99-106

${ }^{6}$ Madan AK, Long AE, Weldon CB, Jaffe BM. Esophageal carcinosarcoma. J Gastrointest Surg 2001; 5: 414-417

\section{Corresponding Author}

\section{Giovannini, M.D.}

Paoli Calmettes Institute 232 boulevard Sainte Marguerite BP 156, 13273 Marseille Cedex 9, France

Fax: $\quad$ +33-4-91223650

E-mail: hdjchir@marseille.fnclcc.fr 